\title{
Characterization of Linear Reduced Order Building Models Using Bode Plots
}

\author{
Moritz Lauster ${ }^{1} \quad$ Dirk Müller $^{1}$ \\ ${ }^{1}$ Institute for Energy Efficient Buildings and Indoor Climate, E.ON Energy Research Center, RWTH Aachen \\ University, Germany mlauster@eonerc.rwth-aachen. de
}

\begin{abstract}
Simulations of energy supply systems on the urban scale call for dedicated thermal building models with low simulation times and still considering relevant dynamic effects. A common approach for such models are reduced order thermal networks that model heat transfer and storage via thermal resistances and capacitances. To contribute to the open question, how much wall elements should be used in such approaches, this paper characterizes and compares four different model topologies with one, two, three and four wall elements. The characterization using the Linear Analysis toolbox in Modelica and Bode plots in Python reveals a significantly different behavior of the One-Element-Model compared to the higher order models. In consequence, the Two-Elements-Model with comparably low simulation times and a similar behavior as the higher order models qualifies for urban scale simulations.
\end{abstract}

Keywords: Modelica, Reduced Order Model, Urban Building Energy Model, Bode plot, Linear Analysis Toolbox

\section{Introduction}

In the context of global warming and anthropogenic greenhouse gas emissions, innovative energy supply systems play an important role to increase energy efficiency. In particular, when supplying entire districts with heat, this calls for sophisticated dynamic building models to consider heat storage effects and compare different system options.

When simulating large numbers of buildings, reasonable simulation times in combination with an appropriate model complexity can be a challenging task. Still, the models need to account for relevant physical effects and details to be able to reflect the buildings' real thermal behavior. In this regard, reduced order models based on thermal networks are an interesting option. They use thermal networks analog to electrical circuits and model heat transfer and storage via thermal resistances and capacitances. The theory of such models is well researched and discussed in Clarke (2001); Davies (2004) and Hensen and Lamberts (2011). The model's complexity and the simulation time is determined by the layout of the network and the number of resistances and capac- itances. Furthermore, the number of resistances and in particular the number of capacitances determine the spatial and physical resolution of the model and thus define the accuracy, with which the dynamics of the building are reproduced. In consequence, the structure of the model needs to be aligned to the simulation task, the resolution of dynamic effects and acceptable simulation times.

Reduced order models account for relatively small simulation times by using a small number of state variables, in the case of thermal networks associated solely to thermal capacitances. In this way, they qualify for urban scale simulations, where uncertainties due to unknown boundary conditions and estimated parameters outweigh modeling accuracy. Still, this leads to the question, what the optimal number of capacitances is for the case of urban scale simulations.

This question calls for a detailed analysis and characterization of the dynamic behavior of promising reduced order modelling options. To do so, Bode plots offer the ability to analyse the magnitude and phase shift of a model output compared to a model input for an entire range of excitation frequencies. They allow a dedicated comparison of different reduced order models for a broad range of frequencies as well as for typical frequencies present in the built environment as e.g. done in Akander (2000) and Ramallo-González et al. (2013). In consequence, Bode plots support finding the optimal number of capacitances, from where adding further elements would not substantially increase model accuracy.

This paper aims at contributing to the field of urban scale simulations by characterizing four different reduced order models using Bode plots. It emphasizes on using Modelica and the Linear Analysis toolbox in combination with a Python-based Bode plot analysis. The next chapter gives an introduction to reduced order building modeling to highlight the impact of thermal capacitances and presents the four investigated model topologies. Afterwards, the paper outlines the setup of the characterization and presents the results using Bode plots to answer the question regarding a reduced order model optimized for urban scale simulations.

\section{Reduced Order Building Models}

As mentioned before, when reducing the order of thermal network models, the question arises, which capacitances 
resp. states are crucial for the dynamic behavior and which can be omitted. Focusing on one thermal zone, the typical entity in building performance simulations, the number of capacitances depends on the number of wall elements and the discretization of these wall elements. This leads to two options to set up reduced order models:

1. Reducing the number of wall elements, e.g. by merging roof, floor and external walls to one wall element.

2. Reducing the number of capacitances per wall element, which represent the discretization of the wall.

The second aspect is already well investigated (e.g. in Davies 1994 and Rouvel and Zimmermann 1997, 1998) and lead to the theory of the periodic depth of penetration, which is standardized in DIN EN ISO 13786 (Deutsches Institut für Normung, 2008a). In consequence, this aspect was found to be of minor importance, so the number is typically fixed to one capacitance per wall element and the capacity depends on the excitation frequency. Two common standardized reduced order models, described in DIN EN ISO 13790 (Deutsches Institut für Normung, 2008b) and VDI 60071 (Verein Deutscher Ingenieure, 2015), follow this approach. However, these two models highlight the differences for the first aspect. While the DIN EN ISO 13790 lumps all walls to one wall element and is explicitly thought for monthly resolution at maximum, the VDI 6007-1 models asymmetrically (external walls) and symmetrically loaded (internal) walls separately. Still, there is no common agreement, how many wall elements are necessary for hourly heat demand calculations and which elements should be lumped.

To contribute to this question, this paper investigates four different model topologies by lumping either all walls to one element (as for DIN EN ISO 13790, Figure 1), distinguish between external and internal walls (as for VDI 6007-1, Figure 2), further divide between walls exposed to solar radiation and floor plates (Figure 3 ) and finally

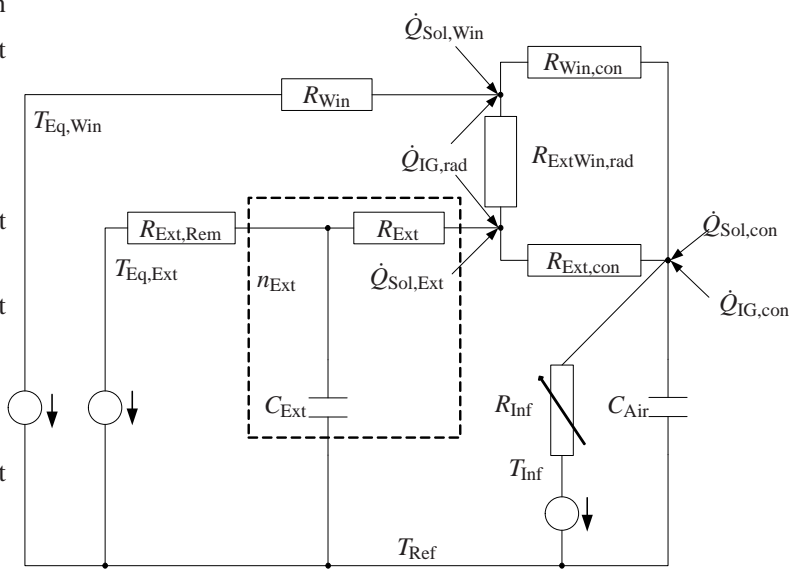

Figure 1. Thermal network of the One-Element-Model from AixLib.

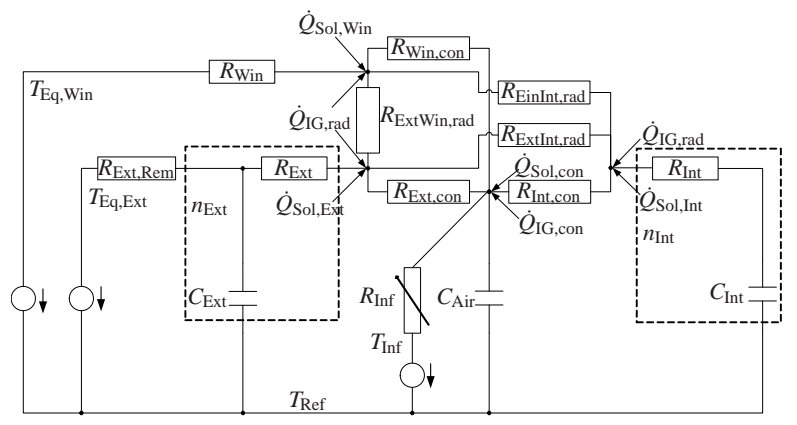

Figure 2. Thermal network of the Two-Elements-Model from AixLib.

separate the roof elements as well (Figure 4). To keep the same basic topology for all options, all models are based on the layout and principles of the VDI 6007-1. Figure 2 represents the original VDI 6007-1 model except for a polygon network instead of a star network for the internal radiation circuit to be able to extend the network without loss of accuracy (as described in Davies 1993).

In addition, heat transfer through windows is handled separately to the external walls, since windows commonly do not incorporate thermal mass and merging windows and walls would lead to a delay in the windows' heat transfer. The heat transfer through windows and heat transfer to the ambient and heat storage in the external walls is handled via resistances and capacitances in the left part of Figure 2. The right part takes care of heat storage in internal walls, while the center part deals with convective and radiative heat exchange within the thermal zone. Both effects are represented by one circuit each, the indoor air temperature can be measured at the star point of the convective circuit. This point connects also to a thermal resistance that is used for infiltration of outdoor air (resp. the associated heat flux) through gaps in the thermal zone's envelope. Further explanations and details about the model can be found in Remmen et al. (2017) and VDI 6007-1 Verein Deutscher Ingenieure (2015).

As mentioned, the One-Element-Model in Figure 1 neglects the differing behavior of internal walls and merges them with external elements to one wall element. With the Two-Elements-Model in Figure 2 in between, the ThreeElements-Model in Figure 3 separates walls exposed to solar radiation and exposed directly to the ground. This follows the assumption that ground coupled wall elements such as floor plates behave thermally different due to the excitation with a very low frequency (with a time constant of about one year). Thus, merging them with elements exposed to solar radiation (excitation with a time constant of one day) might lead to smearing the dynamics of the themal zone. The same argument applies for the FourElements-Model in Figure 4, where the roof is taken care of seperately to the external walls. However, the time constants of roof elements and external walls should be similar, but the excitation is shifted in time for horizontal and vertical elements. Besides the number of state variables, 


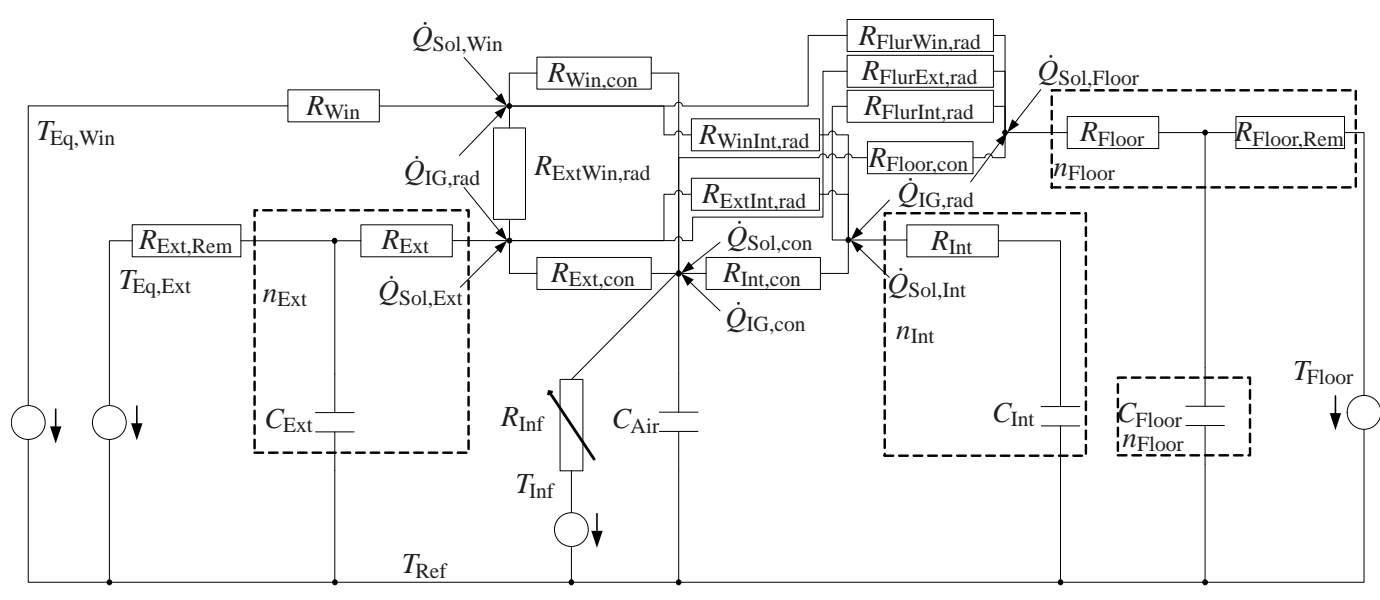

Figure 3. Thermal network of the Three-Elements-Model from AixLib.

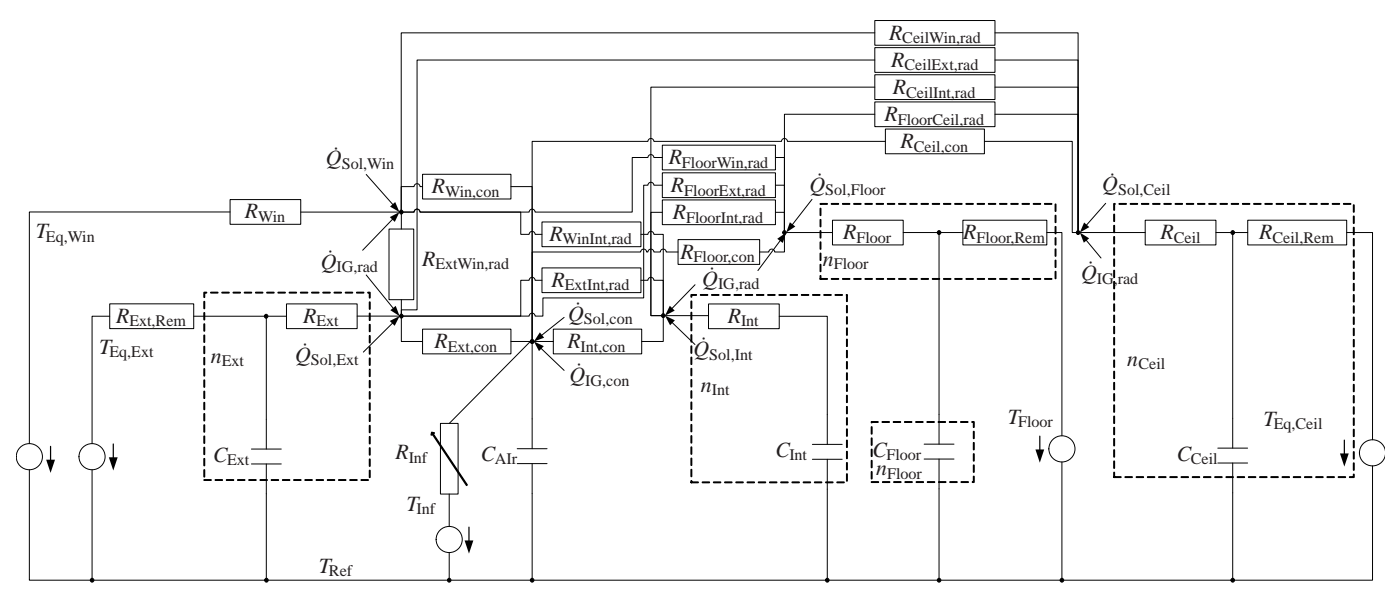

Figure 4. Thermal network of the Four-Elements-Model from AixLib.

simulation time is influenced by the way the physical effects are modelled. In the best case, all phenomena can be modelled with a linear approach, which further lowers simulation time. In the case of reduced order models, there are four effects that can be linearized to obtain a fully linear model.

1. The indoor radiative heat exchange follows the Stefan-Boltzmann law including surface temperatures to the power of four. This can be linearized around a given temperature, usually the zone's set temperature.

2. Convective heat exchange depends on free and forced convection and includes nonlinearities. For typical conditions, different standards (e.g. DIN EN ISO 6946 Deutsches Institut für Normung 2015) provide constant values to linearize the effects.

3. The absorption and transmission of solar radiation on inclined surfaces requires calculation of angles that include nonlinear equations. Since these calculations to not depend on the zone's state variables, all values can be precomputed and serve as inputs to the building model itself.
This setup with linear approaches and a reduced number of state variables leads to models with a relatively low complexity, e.g. for the Two-Elements-Model with 28 unknowns, two state variables and one algebraic loop with constant parameters.

The merging or separation of the building elements leads to four different models with the open question, which model considers all dominant dynamics while neglecting all others to have as small simulation times as possible. For this purpose, all four models need to be characterized in a dynamic way as proposed in the next chapters.

\section{Characterization}

The characterization requires the definition of a use case with a fixed geometry and a known set of physical properties. In this study, the use case is based on a single box-shaped room as the typical layout of a thermal zone. This room follows the geometrical definition of the international validation guideline ASHRAE 140 (ASHRAE, 2007) with a net floor area of $48 \mathrm{~m}^{2}$, two walls with $21.7 \mathrm{~m}^{2}$ and two walls with $16.2 \mathrm{~m}^{2}$ (one internal and one external wall each) as shown in Figure 5. Changes of the geometry do not influence the results, as long as the rela- 


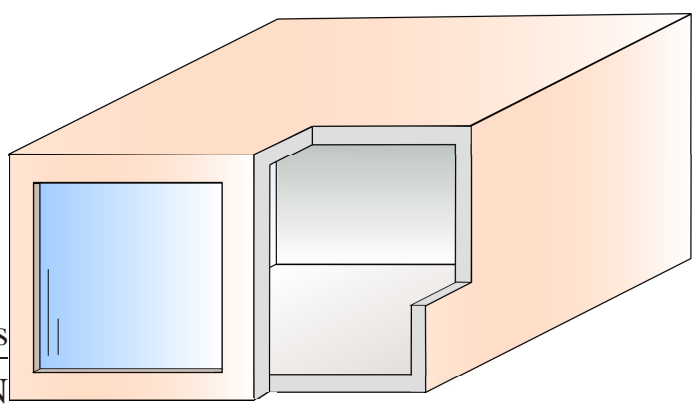

Figure 5. Layout of the test room.

tion between the areas is kept similar. For the given case with similar areas for external walls, roof, floor plate and internal walls, we expect the largest differences between the four models, since all elements have a similar impact.

To cover typical physical properties for the building materials and the wall constructions in the German building stock, the characterization covers 24 setups with insulation levels varying from "EnEV 2009", "EnEV 2002" and "WSchV 1995" to "WSchV 1984", representing German insulation standards of different years. As a second aspect, the building mass is varied from "Light-weight" and "Medium-weight" to "Heavy-weight". Since the cases "EnEV 2009" and "WSchV 1984" turned out to be the extreme cases, the following chapters will focus on these setups.

The use case in all 24 setups and for all four model topologies has been modelled in Modelica using the library AixLib (Müller et al., 2016). AixLib is one of four application libraries based on the same Modelica IBPSA core library, described in Wetter et al. (2015). Both, the AixLib and the IBPSA library, are developed fully opensource and are freely available at https://github. com/RWTH-EBC/AixLib and https://github. com/ibpsa/modelica-ibpsa. The reduced order models are part of the core library and thus of all four application libraries.

To characterize the models using Bode plots, all four model topologies have been transformed to state-space representation of the form

$$
\begin{aligned}
\dot{\mathbf{x}}(t) & =A \mathbf{x}(t)+B \mathbf{u}(t) \\
\mathbf{y}(t) & =C \mathbf{x}(t)+D \mathbf{u}(t)
\end{aligned}
$$

with $\mathbf{x}$ as state vector, $\mathbf{y}$ as output vector and $\mathbf{u}$ as input vector. $A$ stands for the states matrix, $B$ for the input matrix, $C$ for the output matrix and $D$ for the feedthrough matrix. This is a valid approach since we deal with linear time-invariant models. The necessary matrices $A, B, C$ and $D$ can be derived using the ModelicaLinearSystems2.ModelAnalysis.Linearize function of the Linear Analysis toolbox. The created .matfiles containing these matrices can directly be used to create Bode plots in Python with the help of the Python package ModelicaRes, available at https://github. com/kdavies $4 /$ ModelicaRes. In this way, a dedicated analysis of the dynamic behavior of Modelica models is convenient to perform in a semi-automated process.

\section{Results}

This chapter presents the results for the given use case, generated with the process sketched above. As mentioned, it focuses on the setups "EnEV 2009" and "WSchV 1984" as they represent the extreme cases of all setups. The Bode plots show the dynamic behavior for all four model topologies over a frequency range from $10^{-6}$ to $10^{-3} \mathrm{~Hz}$, in particular highlighting typical excitation frequencies in buildings at $1.15^{-5} \mathrm{~Hz}$ (daily) and $2.77^{-4} \mathrm{~Hz}$ (hourly). Since buildings are typically excited by external (outdoor air temperature variations) and internal sources (internal gains, convective and radiative), the Bode analysis is performed twice for each setup. The observed output is the indoor air temperature as the target value. It would be even more benefitial to observe the heat flow through the constructions, which would require strategies to compare the overall heat flows of the different models or observing multiple outputs. These topics are marked as future work and not covered in this paper. The aim is to identify the model with the lowest order that shows no major differences to higher order models for indoor air temperature as output.

Figure 6 shows the Bode plot for external excitation of the heavy-weight "EnEV 2009" setup. The upper part focuses on the magnitude of the output, while the lower part concentrates on the phase shift of the output compared to the input. The pattern of all four models is similar and follows the $\mathrm{PT}_{1}$-behavior of a low-pass filter. The lowpass filter originates from the given RC-element for external wall elements, e.g. shown in Figure 1. In comparison to the other models, only the One-Element-Model reveals some deviations regarding the damping as well as for the phase shift. In particular for the typical daily and hourly excitation frequencies, all models except the One-
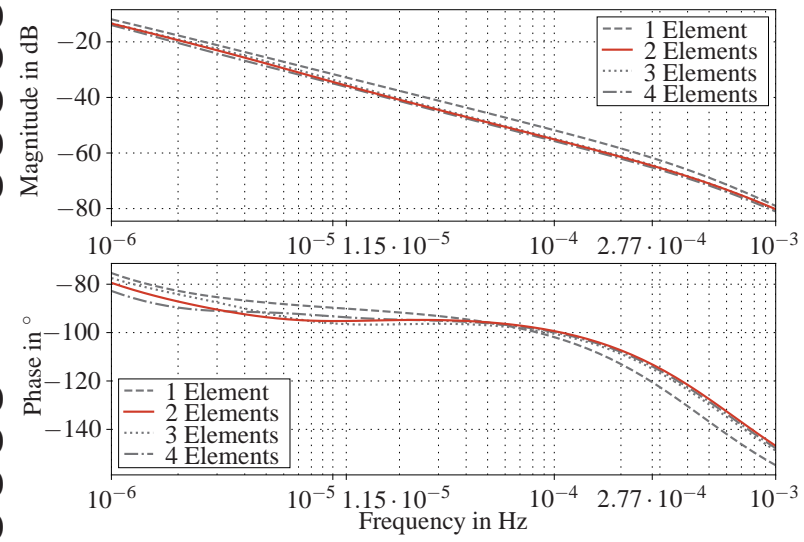

Figure 6. Bode plot for external excitation with heavy-weight setup (EnEV 2009) and indoor temperature as observed output. 

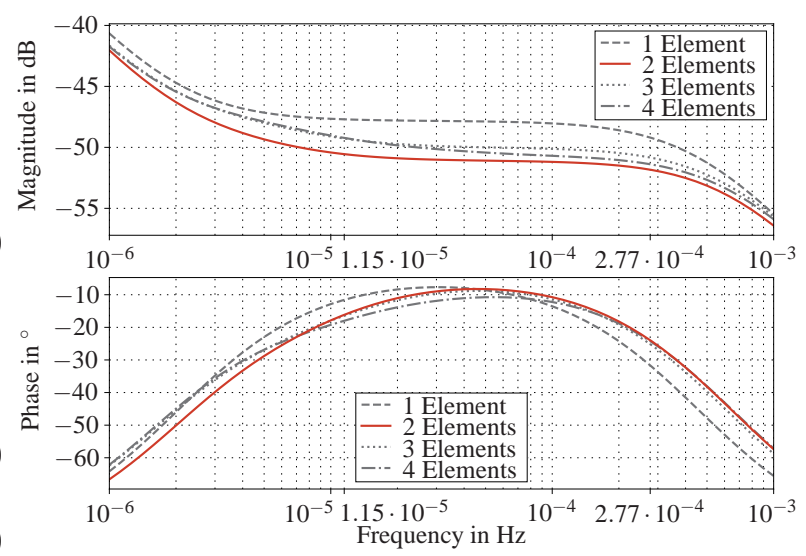

Figure 7. Bode plot for internal excitation with heavy-weight setup (EnEV 2009) and indoor temperature as observed output.

Element-Model behave almost identical.

Figure 7 shows the Bode plot for the same heavy-weight setup in the case of internal excitation. All models show a $\mathrm{PIT}_{1}$-behavior that is related to the interference of the wall elements' PI-behavior and a $\mathrm{PT}_{1}$-behavior of the air volume. In particular the One-Element-Model deviates from the other model topologies and reveals a phase shift in the direction of lower excitation frequencies. This leads to significant differences, especially for an hourly excitation, and can be explained by the missing consideration of the internal walls. In this way, the One-Element-Model on the one hand neglects parts of the zones' thermal mass and other hand does not consider heat transfer between external and internal masses. This is mainly visible for internal excitation, since external excitation is highly damped by the external wall elements. Though, the other three model topologies deviate as well from each other regarding the magnitude as well as the phase shift for low excitation frequencies.

In addition to the heavy-weight setup, Figure 8 shows
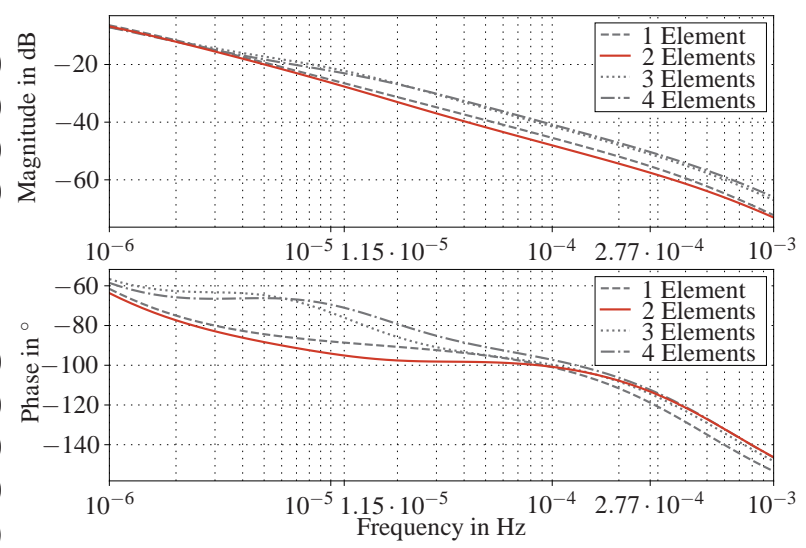

Figure 8. Bode plot for external excitation with light-weight setup (WSchV 1984) and indoor temperature as observed output.
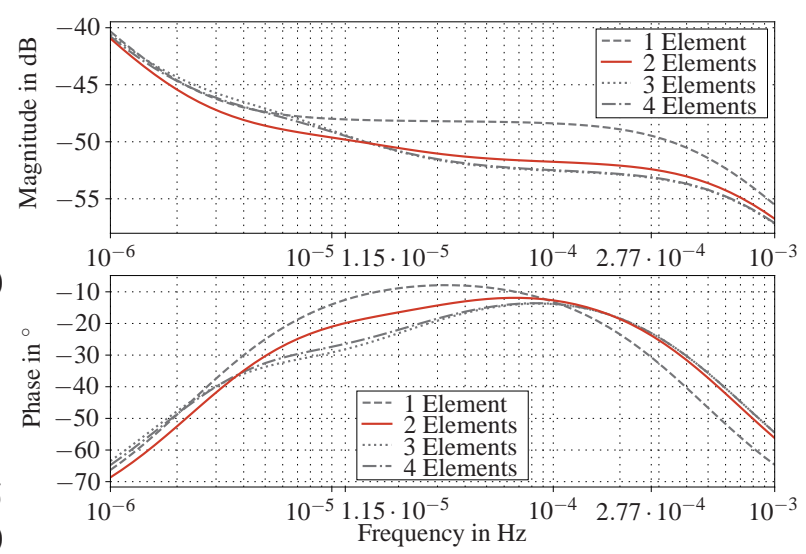

Figure 9. Bode plot for internal excitation with light-weight setup (WSchV 1984) and indoor temperature as observed output.

the Bode plot for external excitation of the light-weight "WSchV 1984" setup. The patterns of all four models are comparable to the behavior in Figure 6, although the Three- and Four-Elements-Model show significant deviations compared to the One- and Two-Elements-Model. The impact of these deviations is hard to assess at this point and will be further investigated in Figure 10.

To complete the set of Bode plots, Figure 9 shows the plot for internal excitation of the light-weight setup. The discussed deviations of the One-Element-Model occur in this plot in an amplified manner. For the magnitude as well as for the phase shift, the One-Element-Model clearly deviates from the other topologies. Two-, Three- and FourElement-Model show similar deviations as in Figure 7.

Resulting from the Bode plots, the Two-, Three- and Four-Elements-Model predominantly show a similar behavior, in particular for typical excitations with daily and hourly time constants. All models have a similar DCgain of approximately 1 , varying between the models at the 10th decimal place. Solely the One-Element-Model reveals major deviations from the other models, in partic-

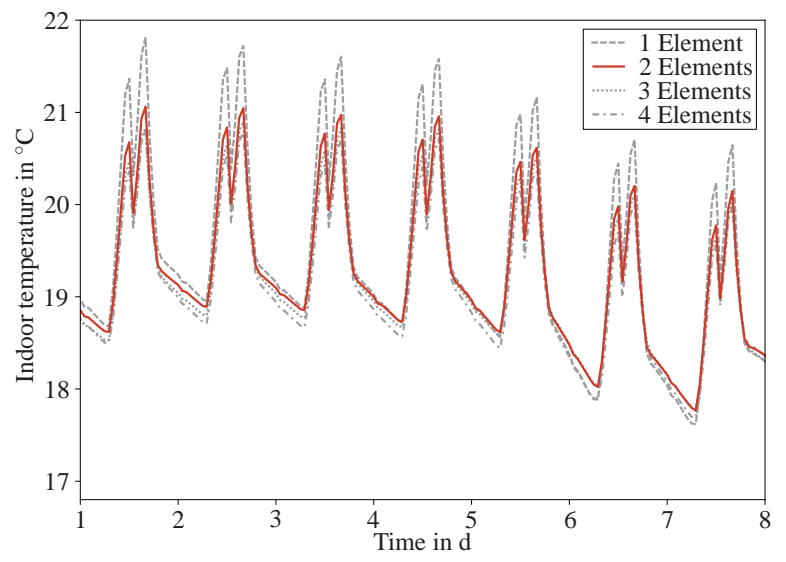

Figure 10. Comparison of all four model topologies in the time domain for the medium-weight setup (EnEV 2002). 
Table 1. Maximum and mean deviation of the indoor temperature in $\mathrm{K}$ between two- and four-elements-model for setups "EnEV 2009 Heavy", "EnEV 2002 Medium" and "WSchV 1984 Light".

\begin{tabular}{lrrr}
\hline & Heavy & Medium & Light \\
\hline Max & 0.3 & 0.4 & 0.7 \\
\hline Mean & 0.1 & 0.1 & 0.1 \\
\hline
\end{tabular}

ular for internal excitation. The deviations in general tend to rise from heavy-weight to light-weight setups and majorly occur for internal excitation. To evaluate the impact of these deviations, it is necessary to complement the analyses in the frequency domain by investigations in the time domain.

For this, yearly simulations of all four model topologies are performed with time-dependent weather data (TRY 2010 data for Mannheim, Germany), varying internal gains (generic profiles for persons and machines, convective and radiative) and free-floating indoor air temperature. Figure 10 shows seven days in spring, where typical indoor air temperatures between 17 and $21^{\circ} \mathrm{C}$ occur and the typical excitation frequencies of one hour and one day can be observed. For reasons of clarity, the figure focuses on the medium-weight setup "EnEV2002". The figure shows a significant overshoot of the One-Element-Model in times of local maximum temperatures. This correlates with the observations in Figure 7 and Figure 9, where the One-Element-Model shows a lower damping of internal excitations. This behavior leads to maximal differences of the indoor air temperature of $1.2 \mathrm{~K}$ compared to the other model topologies. In comparison, the difference between Two- and Four-Elements-Model for the same setup is $0.4 \mathrm{~K}$, as given in Table 1 . The differences between the higher-order models tend to rise from heavy-weight to light-weight setups.

Given the difference in the frequency as well as in the time domain between the One-Element-Model and the other topologies, this approach with only one state variable for the wall elements seems inappropriate for dynamic builing performance simulations. Following the approach to keep the number of states as small as possible, the Two-Elements-Model comes into focus and shows a significantly better behavior. With these results at hand, the Two-Elements-Model qualifies for dynamic heat demand calculations on urban scale, where simulation time plays a major role and modelling simplifications are outweighed by uncertainties of the boundary conditions.

\section{Conclusions}

Urban scale simulations of large building stocks for energy efficient supply systems call for dynamic building models with low simulation times while accepting modelling simplifications. Such simplifications are typically outweighed by uncertainties in boundary conditions on ur- ban scale. A common approach for such building models are reduced order models based on thermal networks, which represent heat transfer and storage via thermal resistances and capacitances. The models' simulation times, complexity and order is correlated to the number of state variables resp. thermal capacitances. Although this topic has been well researched and standardized approaches exist on single wall level, the questions remains, how many wall elements are necessary to model heat storage effects on urban scale in a sufficient way.

This paper contributes to this question by characterizing four model topologies with one, two, three and four wall elements. The thermal masses of a given use case are therefore merged all together, separated into internal and external masses, additionally with a separate element for the floor plate or further splitted up to a separate roof element. The use case is a simple, box-shaped room, which is commonly used for model validation. To take into account different insulation and building mass levels, the use case contains 24 setups from light-weight, well insulated scenarios up to heavy-weight, hardly insulated versions. As extreme cases, the characterization focuses on an heavy-weight "EnEV 2009" and on a light-weight "WSchV 1984" setup.

All model topologies and setups are modelled using the Modelica library AixLib, https://github. com/RWTH-EBC/AixLib, an application library of the IBPSA core library, https: //github. com/ibpsa/ modelica-ibpsa.

The characterization makes use of Bode plots and comparisons in the time domain to analyse the behavior of all four topologies with regard to magnitude and phase shift of the indoor air temperature compared to a chosen input over a range of excitation frequencies. Typical excitation frequencies in the field of building performance simulation with time constants of one day and one hour can in this way clearly be highlighted. The necessary matrices to describe the model's transfer function can be obtained with the ModelicaLinearSystems 2.ModelAnalysis.Linearize function out of the Linear Analysis toolbox. The resulting files can directly be used to create dedicated Bode plots in Python with the help of the Python package ModelicaRes, https://github. $\mathrm{com} / \mathrm{kdavies} 4 /$ ModelicaRes.

The results show that the behavior of the One-ElementModel significantly differs from the higher order models, for the magnitude as well as the phase shift, when observing the indoor air temperature while exciting outdoor air temperature or internal gains. This originates in neglecting internal masses, what leads to a significantly different transfer function.The Two-, Three- and Four-ElementsModel show slight differences in the Bode plots, what requires further analyses in the time domain. The simulation of one year reveals maximal differences in the freefloating indoor air temperature between Two- and FourElements-Model of $0.4 \mathrm{~K}$. The same case shows differences between One-Element-Model and higher order 
models of $1.2 \mathrm{~K}$.

Based on these results, the Two-Elements-Model qualifies for urban scale simulations with low simulation times while keeping a similar behavior compared to higher order models. As the differences partly depend on the insulation and thermal mass level, further research should result in an adaptive method to automatically choose a reduced order modelling approach based on these properties.

\section{Acknowledgements}

We gratefully acknowledge the financial support by BMWi (German Federal Ministry of Economic Affairs and Energy) under promotional reference 03ET1562A.

\section{References}

Jan Akander. The ORC method: Effective modelling of thermal performance of multilayer building components. Dissertation, The Royal Institute of Technology, Stockholm, 2000.

ASHRAE. Standard Method of Test for the Evaluation of Building Energy Analysis Computer Programs, 2007.

J. A. Clarke. Energy simulation in building design. ButterworthHeinemann, Oxford, 2. ed edition, 2001. ISBN 0750650826.

M. G. Davies. Definitions of Room Temperature. Building and Environment, 28(4):383-398, 1993. doi:10.1016/0360-1323(93)90015-U.

M. G. Davies. The Thermal Response of an Enclosure to Periodic Excitation: The CIBSE Approach. Building and Environment, 29(2):217-235, 1994.

Morris G. Davies. Building Heat Transfer. John Wiley \& Sons, Hoboken, NJ, 2004. ISBN 978-0-470-84731-2.

Deutsches Institut für Normung. Thermal performance of building components - Dynamic thermal characteristics - Calculation methods, 2008a.

Deutsches Institut für Normung. Energy performance of buildings - Calculation of energy use for space heating and cooling, 2008b.

Deutsches Institut für Normung. Building components and building elements - Thermal resistance and thermal transmittance - Calculation method, 2015.

Jan Hensen and Roberto Lamberts, editors. Building performance simulation for design and operation. Spon Press, London and New York, NY, 2011. ISBN 978-0-415-47414-6.

Dirk Müller, Moritz Lauster, Ana Constantin, Marcus Fuchs, and Peter Remmen. AixLib - An Open-Source Library within the IEA-EBC Annex60 Framework. In BauSIM 2016: Sixth German-Austrian IBPSA Conference, pages 3-9, 2016.

Alfonso P. Ramallo-González, Matthew E. Eames, and David A. Coley. Lumped parameter models for building thermal modelling: An analytic approach to simplifying complex multilayered constructions. Energy and Buildings, 60:174-184, 2013. ISSN 03787788. doi:10.1016/j.enbuild.2013.01.014.
Peter Remmen, Moritz Lauster, Michael Mans, Marcus Fuchs, Tanja Osterhage, and Dirk Müller. TEASER: An open tool for urban energy modelling of building stocks. Journal of Building Performance Simulation, pages 1-15, 2017. doi:10.1080/19401493.2017.1283539.

Lothar Rouvel and Frank Zimmermann. Ein regelungstechnisches Modell zur Beschreibung des thermisch dynamischen Raumverhaltens. HLH Lüftung/Klima - Heizung/Sanitär - Gebäudetechnik, 48,49(10, 12, 1):66-75, 24-31,18-29, 1997, 1998.

Verein Deutscher Ingenieure. Berechnung des instationären thermischen Verhaltens von Räumen und Gebäuden - Raummodell, 2015.

Michael Wetter, Marcus Fuchs, Pavel Grozman, Lieve Helsen, Filip Jorissen, Moritz Lauster, Dirk Müller, Christoph Nytsch-Geusen, Damien Picard, Per Sahlin, and Matthis Thorade. IEA EBC Annex 60 Modelica Library - An International Collaboration to Develop a Free Open-Source Modelica Library for Buildings and Community Energy Systems. In Building Simulation 2015: 14th Conference of International Building Performance Simulation Association, pages 395-402, 2015. 
